\title{
Hyperprogressive Disease and Early Hypereosinophilia After Anti-PD-1 Treatment: A Case Report
}

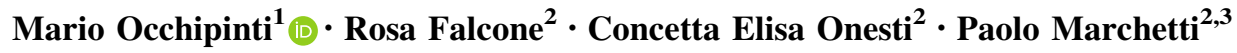

Published online: 13 March 2018

(C) The Author(s) 2018

\begin{abstract}
Hyperprogressive disease (HPD) has been recently proposed as a new pattern of progression in patients treated with immune checkpoint inhibitors (ICIs). Until now, no biological marker has been found to predict this accelerated tumour growth. We describe the case of a 62-year-old women who experienced a marked increase in absolute eosinophil count (AEC) concurrently with a huge radiological progression after the first nivolumab dose in absence of other immune-related adverse events (irAEs). Further investigations are needed to establish the role of early hypereosinophilia as a marker of progression and to identify patients who might not benefit from ICI treatment.
\end{abstract}

Mario Occhipinti

mario.occhipinti@uniroma1.it

1 Medical Oncology Unit B, Policlinico Umberto I, "Sapienza" University of Rome, Viale Regina Elena 324, 00161 Rome, Italy

2 Medical Oncology Unit, Sant' Andrea Hospital, "Sapienza" University of Rome, Rome, Italy

3 Istituto Dermopatico dell'Immacolata-IRCCS, Rome, Italy

\section{Key Points}

Hyperprogressive disease (HPD) is a novel pattern of response during immune checkpoint inhibitor (ICI) treatment. Immune-related eosinophilia is a biological immune-related adverse effect with antiprogrammed death 1 (anti-PD-1) or antiprogrammed death ligand 1 (anti-PD-L1).

In this case report, anti-PD-1 administration resulted in a marked increase in absolute eosinophil count (AEC) concurrently with a huge radiological progression.

Early hypereosinophilia may be a negative predictive factor of ICIs treatment.

\section{Introduction}

Anti-programmed death 1 (anti-PD-1) and anti-programmed death ligand 1 (anti-PD-L1) monoclonal antibodies (mAbs) are immune checkpoint inhibitors (ICIs) capable of restoring immunity against tumours and improving survival in several tumour types [1]. Nevertheless, some patients seem to not benefit from immunotherapy and, indeed, it seems that ICIs accelerate the drive towards progression. In fact, Champiat et al. documented a new pattern of rapid progression in patients treated with ICIs: hyperprogressive disease (HPD). It is defined as a RECIST (Response Evaluation Criteria In Solid Tumors) progression at the first evaluation and as a $\geq 2$-fold 
increase of the tumour growth rate (TGR) between the reference (prior to treatment onset) and the experimental (between baseline and the first tumour evaluation) periods [2]. Kato et al. reported that patients with mouse double minute 2 homolog (MDM2) family amplifications appear to be at risk of accelerated progression after immunotherapy [3]. At present, biomarkers for outcome during immunecheckpoint blockade are not available and further research is strongly needed as these may influence individual treatment choice. Absolute eosinophil count (AEC) has been recently investigated as a new potential biomarker of outcome in patients affected by melanoma. An increase in the eosinophil count has been correlated with an improved overall survival in metastatic melanoma patients treated with ipilimumab, and recently it has been studied in patients during anti-PD-1/anti-PD-L1 treatment [4-8]. Bernard-Tessier et al. proposed immune-related eosinophilia as a new biological immune-related adverse effect with anti-PD-1 or anti-PD-L1 [8]. It has been defined as a

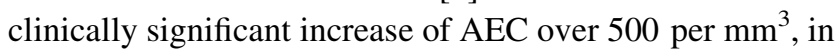
two samples at different times, during anti-PD1 or anti-PDL1 treatment. We describe here the first case of a patient who experienced dramatic hyperprogressive disease and early elevation of AEC after the first dose of anti-PD-1 treatment.

\section{Case Report}

In March 2015, a 60-year-old Caucasian female never smoker presented to the emergency department with fever and persistent cough. The chest X-ray and the subsequent CT scan showed a hilar lung mass on the upper left lobe, multiple hilar, para-tracheal, subcarinal and prevascular lymphadenopathies, bone pelvis and vertebral lesions. The histological examination, obtained by bronchoscopy, revealed a non-small-cell lung cancer (NSCLC), CK7+ and TTF1+ adenocarcinoma, EGFR wt, $P D-L 1$ negative, $A L K$ and ROS1 not rearranged. She was staged as cT3N3M1b (stage IV) according to the American Joint Committee on Cancer Staging Manual, 7th edition [9].

From April 2015 to August 2015, the patient underwent first-line platinum $75 \mathrm{mg} / \mathrm{m}^{2}$ plus pemetrexed $500 \mathrm{mg} / \mathrm{m}^{2}$ chemotherapy given every 3 weeks for six cycles. The CT scan performed on August 2015 revealed a complete response on the lung mass and on lymphadenopathies and a bone stable disease. The patient continued maintenance treatment with pemetrexed until July 2016 when the surveillance CT scan showed an oligoprogression on the right adrenal gland with a $4-\mathrm{cm}$ mass (SUV 25). In September 2016, the patient underwent right adrenalectomy (histological examination: NSCLC metastases) and continued maintenance pemetrexed until November 2016.
The CT scan performed on December 2016 revealed disease progression on bone metastases, lymphadenopathies and a recurrence on the adrenalectomy site.

The patient started second-line treatment with docetaxel $75 \mathrm{mg} / \mathrm{m}^{2}$ plus nintedanib $200 \mathrm{mg}$ twice a day every 3 weeks. She experienced AST/ALT grade 2 (G2) elevation and neutropenia grade $1(\mathrm{G} 1)$. After the third cycle, the CT scan showed disease progression with new abdominal lymphadenopathies.

Administration of nivolumab $3 \mathrm{mg} / \mathrm{kg}$ every 2 weeks was started on 29 March 2017 and the patient had a good clinical status. One week after the first dose, laboratory tests revealed a marked increase in AEC of $10.7 \times 10^{3}$ $(0.0-0.8)$ and moderate anaemia $(\mathrm{Hb} 8.4 \mathrm{~g} / \mathrm{dL})$. There were no other perturbations in laboratory tests (blood chemistry and endocrinological functions). The patient experienced fatigue (G1), no fever, no rash or any other immune-related adverse events (irAEs); stool sample was negative for parasites and heart evaluation was negative for any damage. A thorax CT scan, performed in order to exclude an eosinophilic pneumonia, revealed a lung recurrence on the upper left lobe $(1.2 \times 2 \mathrm{~cm})$. The patient continued clinical observation and received the second nivolumab dose on 12 April 2017. A week later, the patient developed progressive fatigue and appetite loss; AEC was still increasing $\left(12.3 \times 10^{3}\right)$. The patient was hospitalized for severe anaemia and worsening clinical status. A CT scan was performed on 27 April with evidence of a further increase of the lung mass on upper left lobe $(7.2 \times 2.7 \mathrm{~cm}$ vs $1.2 \times 2 \mathrm{~cm}$ ), pleural effusion, several liver metastases and peritoneal carcinomatosis; because of the severe rapidly progressive anaemia, a gastroscopy was carried out showing multiple neoplastic gastric ulcerative lesions (Fig. 1). Despite the AEC decreasing $\left(1.1 \times 10^{3}\right)$, nivolumab treatment was discontinued as a result of clinical deterioration and radiological progression disease. The patient died 2 months after the last nivolumab dose.

\section{Discussion}

Hyperprogressive disease (HPD) has been observed in $9 \%$ of patients treated with anti-PD-1/PD-L1 [2]. It is associated with older age ( $\geq 65$ years old) and worse overall survival. Tumour disease growth acceleration could be linked to the blockade of $P D-1 / P D-L 1$ signalling in combination with genetic cell alterations such as $M D M 2$ family amplification. Kato et al. identified, among 155 patients during treatment with ICIs, a time-to-treatment failure (TTF) $<2$ months in all six patients with MDM2/MDM4 amplification [3]. Moreover, mechanisms of adaptive resistance and tumour escape such as up-regulation of alternative immune checkpoints have been observed [10]. 


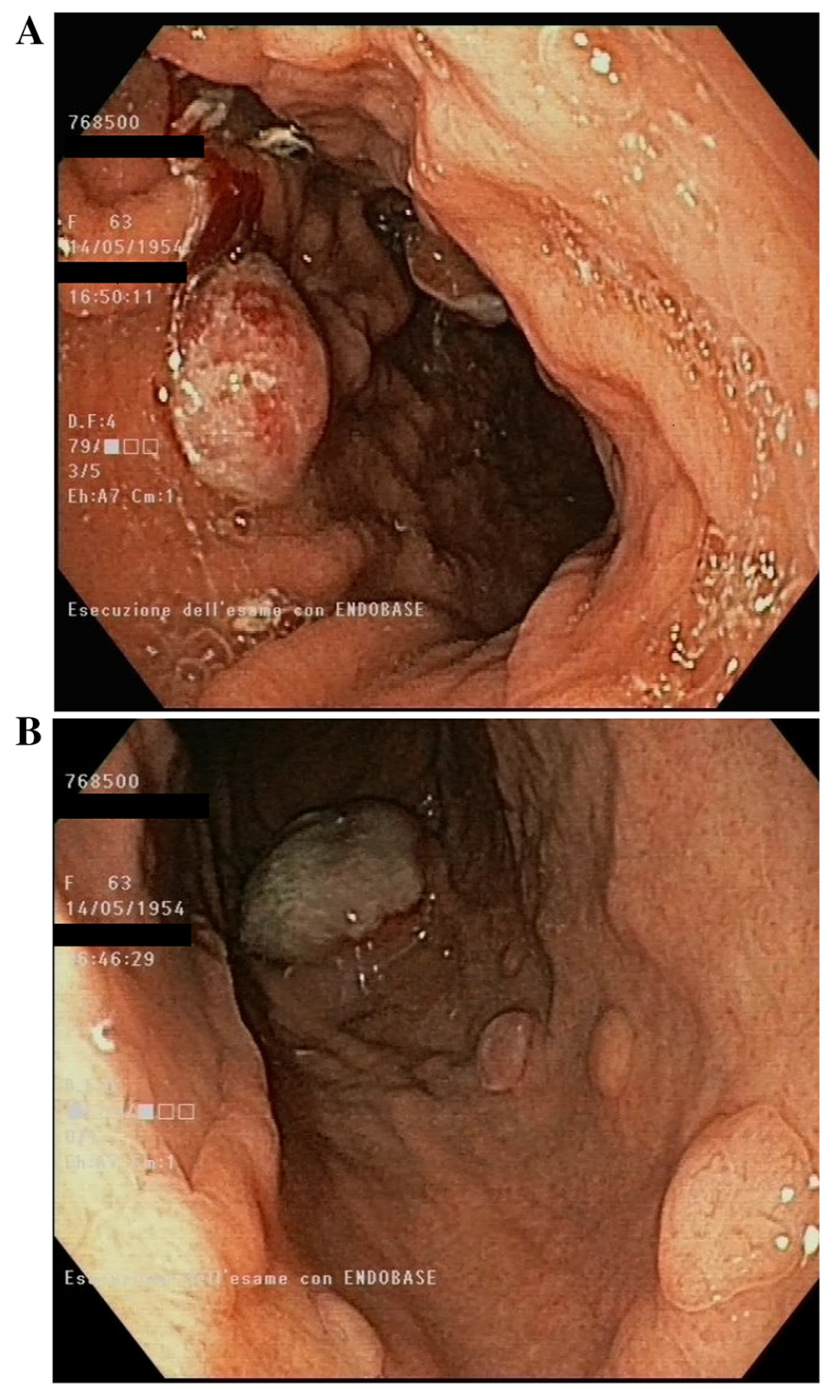

Fig.1 Endoscopic findings of multiple neoplastic gastric ulcerative lesions

In literature, increase in AEC during treatment with ICIs has been reported as a favourable biomarker in metastatic melanoma patients [6, 7]. Recently, Bernard-Tessier et al. reported the first case series of immune-related eosinophilia during anti-PD-1/PD-L1 treatment, showing kinetics leucocyte changes restricted to the eosinophils lineage [8]. ICIs can lead to interferon (IFN)- $\gamma$ elevation, which in turn activates $J A K$-STAT signalling, leading to the production of interferon-inducible chemokines [11, 12]. In particular, activation of the STATI pathway by IFN- $\gamma$, associated with the signalling triggered by tumour necrosis factor (TNF), is responsible for the induction of IFN-inducible chemokines CXCL9, CXCL10 and CXCL11 attracting TH1 cells [13]. In an animal tumour model study, authors observed that tumour-infiltrating eosinophils, activated by TH1 response, secrete chemokines, guiding $\mathrm{CD} 8+\mathrm{T}$ cells to the tumour, resulting in tumour rejection and prolonged survival [14]. In addition, IFN- $\gamma$ inhibits STAT6 signalling, influencing the $\mathrm{TH} 2$ response $[13,15]$. The presence of an activated $\mathrm{TH} 2$ eosinophil response could sustain M2-like macrophages in tumours and promote an immunosuppressive and pro-tumour environment [16]. Hence, the activation and polarization of eosinophil response could predict tumour growth or tumour rejection.

In our case, the patient experienced dramatic hyperprogressive disease after the first dose of nivolumab according to Kato's criteria, as TTF $<2$ months, $>50 \%$ increase in tumour burden compared with pre-immunotherapy imaging and $>2$-fold increase in progression pace (Fig. 2). Concurrently, she reported a marked increase in AEC. At that time, there was no clinical evidence of allergic manifestation, signs of parasitic infection, or other irAEs. No other medications were started before or after the first perfusion of nivolumab. Conversely to literature findings, we observed a negative association between the increase in AEC and the outcome.

\section{Conclusions}

In summary, this case reports an early eosinophilia after the first nivolumab infusion, spontaneously recovered. It occurred simultaneously with clinical deterioration of the patient and the radiologic finding of hyperprogressive
Fig.2 Changes in absolute eosinophil counts during nivolumab treatment. Arrows show nivolumab dose administration
EOSINOPHILS

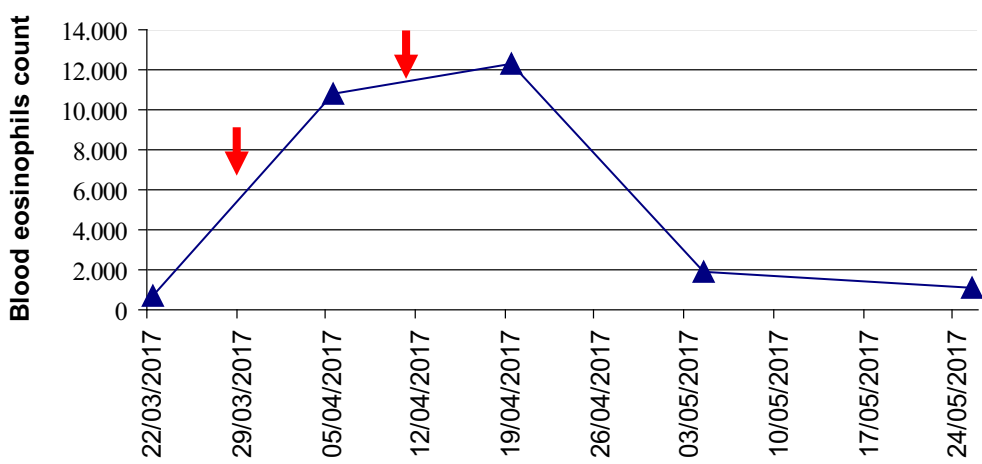

—EOSINOPHILS 
disease. No other medical conditions could explain the aetiology of hypereosinophilia and only the time relation with nivolumab administration may suggest the causality. We can't demonstrate the link between the early hypereosinophilia and the pattern of hyperprogression. Prospective studies are needed to determine the precise role of changes in AEC during treatment with ICIs and to clarify if the early presentation of hypereosinophilia may be a predictive factor of ICIs failure and of radiological and clinical hyperprogression.

\section{Compliance with Ethical Standards}

Funding Mario Occhipinti, Rosa Falcone, Concetta Elisa Onesti and Paolo Marchetti declare that they have no sources of funding to disclose.

Conflicts of interest Mario Occhipinti, Rosa Falcone, Concetta Elisa Onesti and Paolo Marchetti declare that they have no conflicts of interest to disclose.

Ethical approval All procedures were in accordance with the 1964 Helsinki Declaration (and its amendments). No approval by ethical committee or institutional review board was required.

Informed consent The patient died in June 2017. Written informed consent was obtained from the husband of the patient for publication of the case.

Open Access This article is distributed under the terms of the Creative Commons Attribution-NonCommercial 4.0 International License (http://creativecommons.org/licenses/by-nc/4.0/), which permits any noncommercial use, distribution, and reproduction in any medium, provided you give appropriate credit to the original author(s) and the source, provide a link to the Creative Commons license, and indicate if changes were made.

\section{References}

1. Lipson EJ, Forde PM, Hammers HJ, Emens LA, Taube JM, Topalian SL. Antagonists of PD-1 and PD-L1 in cancer treatment. Semin Oncol. 2015;42(4):587-600.

2. Champiat S, Dercle L, Ammari S, et al. Hyperprogressive disease (HPD) is a new pattern of progression in cancer patients treated by anti-PD-1/PD-L1. Clin Cancer Res. 2017;23(8):1920-8.
3. Kato S, Goodman A, Walavalkar V, Barkauskas DA, Sharabi A, Kurzrock R. Hyper-progressors after immunotherapy: analysis of genomic alterations associated with accelerated growth rate. Clin Cancer Res. 2017;23(15):4242-50.

4. Delyon J, Mateus C, Lefeuvre D, et al. Experience in daily practice with ipilimumab for the treatment of patients with metastatic melanoma: an early increase in lymphocyte and eosinophil counts is associated with improved survival. Ann Oncol. 2013;24(6):1697-703.

5. Moreira A, Leisgang W, Schuler G, Heinzerling L. Eosinophilic count as a biomarker for prognosis of melanoma patients and its importance in the response to immunotherapy. Immunotherapy. 2017;9(2):115-21.

6. Weide B, Martens A, Hassel JC, et al. Baseline biomarkers for outcome of melanoma patients treated with pembrolizumab. Clin Cancer Res. 2016;22(22):5487-96.

7. Gaba L, Victoria I, Pineda E, et al. Changes in blood eosinophilia during anti-PD1 therapy as a predictor of long term disease control in metastatic melanoma. ASCO Meet Abstr. 2015;33(15_suppl):9069.

8. Bernard-Tessier A, Jeanville $P$, Champiat $S$, et al. Immune-related eosinophilia induced by anti-programmed death 1 or deathligand 1 antibodies. Eur J Cancer. 2017;81:135-7.

9. Edge SB, Byrd DR, Compton CC, Frits AG, Greene FL, Trotti A. AJCC cancer staging manual. 7th ed. New York: Springer; 2010.

10. Koyama S, Akbay EA, Li YY, et al. Adaptive resistance to therapeutic PD-1 blockade is associated with upregulation of alternative immune checkpoints. Nat Commun. 2016;7:10501.

11. Peng W, Liu C, Xu C, et al. PD-1 blockade enhances T-cell migration to tumors by elevating IFN- $\gamma$ inducible chemokines. Cancer Res. 2012;72(20):5209-18.

12. Schindler C, Levy DE, Decker T. JAK-STAT signaling: from interferons to cytokines. J Biol Chem. 2007;282(28):20059-63.

13. Liu LY, Bates ME, Jarjour NN, Busse WW, Bertics PJ, Kelly EA. Generation of Th1 and Th2 chemokines by human eosinophils: evidence for a critical role of TNF- $\alpha$. J Immunol. 2007;179(7):4840-8.

14. Carretero R, Sektioglu IM, Garbi N, Salgado OC, Beckhove P, Hämmerling GJ. Eosinophils orchestrate cancer rejection by normalizing tumor vessels and enhancing infiltration of CD8+ T cells. Nat Immunol. 2015;16(6):609-17.

15. Huang Z, Xin J, Coleman J, et al. IFN- $\gamma$ suppresses STAT6 phosphorylation by inhibiting its recruitment to the IL-4 receptor. J Immunol. 2005;174(3):1332-7.

16. Wu D, Molofsky AB, Liang HE, et al. Eosinophils sustain adipose alternatively activated macrophages associated with glucose homeostasis. Science. 2011;332(6026):243-7. 